\title{
Investigation of condensation shocks and re-entrant jet dynamics in a cavitating nozzle flow by Large-Eddy Simulation
}

\author{
Theresa Trummler*, Steffen J. Schmidt, Nikolaus A. Adams \\ Chair of Aerodynamics and Fluid Mechanics, Technical University of Munich \\ Boltzmannstr. 15, 85748 Garching bei München, Germany
}

\begin{abstract}
Cloud cavitation is related to an intrinsic instability where clouds are shed periodically. The shedding process is initiated either by the motion of a liquid re-entrant jet or a condensation shock. Cloud cavitation in nozzles interacts with the flow field in the nozzle, the mass flow and the spray break-up, and causes erosion damage. For nozzle geometries cloud shedding and the associated processes have not yet been studied in detail.

In this paper, we investigate the process of cloud cavitation shedding, the re-entrant jet and condensation shocks in a scaled-up generic step nozzle with injection into gas using implicit Large-Eddy Simulations (LES). For modeling of the cavitating liquid we employ a barotropic equilibrium cavitation model, embedded in a homogeneous multi-component mixture model. Full compressibility of all components is taken into account to resolve the effects of collapsing vapor structures.

We carry out simulations of two operating points exhibiting different cavitation regimes. The time-resolved, three-dimensional simulation results cover several shedding cycles and provide deeper insight into the flow field. Our results show that at lower cavitation numbers, shedding is initiated by condensation shocks, which has not yet been reported for nozzle flows with a constant crosssection. We analyze the cavitation dynamics and the shedding cycles of both operating points. Based on our observations we propose modifications to established schematics of the cloud shedding process. Additionally, we analyze the near-wall upstream flow in and underneath the vapor sheet and possible driving mechanism behind the formation of the re-entrant jet.
\end{abstract}

Keywords: Large-Eddy Simulation; cavitation dynamics; schematics of cloud shedding processes; re-entrant jet; condensation shock; nozzle

\section{Introduction}

Cavitation phenomena in nozzles play a crucial role for various hydraulic systems such as dump throttles of liquid propellant rocket engines, orifices of hydraulic jacks and injector components. For the latter, cavitation can affect mass flow (Nurick, 1976, Payri et al., 2004, Trummler et al. 2018), spray break up (Örley et al. 2015; Reitz and Bracco, 1982; Sou et al., 2007) and resilience against cavitation erosion (Asi, 2006). Understanding of cavitation dynamics and of underlying mechanisms of cavitation in injector nozzles is essential.

\footnotetext{
${ }^{*}$ Corresponding author

Email address: theresa.trummler@tum.de (Theresa Trummler)
} 
Cloud cavitation is a form of partial cavitation where vapor clouds periodically shed from the main cavity (Laberteaux and Ceccio, 2001; Reisman et al., 1998). This form of cavitation occurs in flows around bodies, such as hydrofoils (De Lange and De Bruin, 1998; Kubota et al., 1989; Le et al., 1993), and in internal flows, e.g. Venturi nozzles (Hayashi and Sato, 2014; Rudolf et al., 2014), converging-diverging ducts with a rectangular cross-section (wedges) (Ganesh et al., 2016, Stutz and Reboud, 1997), and other types of orifices and nozzles (He et al., 2016; Saito and Sato, 2003; Sato and Saito, 2002; Sou et al., 2007; Stanley et al., 2011, 2014; Sugimoto and Sato, 2009). The periodic cloud shedding process is initiated by the upstream motion of a disturbance, which can be either a thin liquid film underneath the cavity (re-entrant jet) or a bubbly condensation shock.

A re-entrant jet characterizes the motion of a liquid jet underneath the fixed cavity in upstream direction and is generally considered to initiate the shedding process. Le et al. (1993) proposed a schematic description for the periodic cloud shedding process on hydrofoils, which Stanley et al. (2014) adapted for internal nozzle flows. After the collapse of a shed cloud, a re-entrant jet develops at the end of the cavity and travels upstream underneath the cavity. When the re-entrant jet has covered the entire cavity and reached the inception point of the cavity, the cavity is shed and a new one begins to form (Le et al., 1993, Wade and Acosta, 1966). The shed cloud convects downstream with the flow, in a rolling motion (Kubota et al., 1989, Le et al., 1993). In the region of increased pressure downstream the shed cloud collapses, emitting an intense shock wave leading to pressure peaks orders of magnitude larger than the pressure in the mean flow (Reisman and Brennen, 1996 . Reisman et al., 1998), which can cause cavitation erosion (Gopalan and Katz, 2000; Petkovšek and Dular, 2013). Moreover, these cavitation induced pressure fluctuations (Ji et al., 2015; Leroux et al., 2004) are also considered to contribute to the formation of the re-entrant jet, as discussed below.

Although many experimental and numerical studies have investigated re-entrant jets and their decisive role in cloud shedding (e.g. Furness and Hutton (1975); Gopalan and Katz (2000); Kawanam. et al. (1997); Lush and Skipp (1986)), the driving mechanism behind the formation of the jet has not yet been clarified. One theory is that the re-entrant jet is formed due to the stagnation point behind the cavity (at the closure), where the flow surrounding the cavity impinges on the wall. Another suggestion is that the pressure peaks due to the collapse of shed clouds promote the motion of the re-entrant jet (Leroux et al., 2004, 2005). Coutier-Delgosha et al. (2007), however, found no general relation between the shock waves after cloud collapse and the re-entrant jet motion. Callenaere et al. (2001) consider the cavity thickness in relation to the re-entrant jet thickness and the negative pressure gradient to be the two most relevant parameters for the instability of the re-entrant jet.

Several experimental investigations have assessed the velocity of re-entrant jets. For the cavitating flow on a hydrofoil, Pham et al. (1999) measured that the re-entrant jet velocity at the end of the cavity is about the free stream velocity and decreases upstream. Decreasing upstream jet velocity was also observed by Sakoda et al. (2001). Le et al. (1993) found that the velocity of perturbations traveling upstream is close to the magnitude of the free stream flow. Stanley et al. (2014) tracked bubbles in the liquid film underneath the cavity and obtained velocities of $20-30 \%$ of the free-stream velocity, which is a smaller magnitude than reported in previous findings.

In addition to re-entrant jets, condensation shocks can also initiate periodic cloud shedding. The occurrence of condensation shocks was predicted back in 1964 by Jakobsen (1964). Later, condensation shocks on hydrofoils have been studied experimentally by e.g. Arndt et al. (2001); 
Reisman et al. (1998). Converging-diverging geometries have been investigated experimentally by Ganesh et al. (2016); Jahangir et al. (2018); Wang et al. (2017); Wu et al. (2017) and recently by compressible numerical simulations (Budich et al., 2018). For shock initiated shedding, one observes an upstream moving bubbly shock rather than a re-entrant jet. The formation of bubbly shocks is more likely when the cavitation number decreases, and for increased void fraction in the cavity (Ganesh et al., 2016). We are not aware of publications addressing the occurrence of condensation shocks in nozzles with constant cross-sections. However, the observations by Stanley et al. (2014) may be explained by a condensation shock mechanism.

The flow field within realistic geometries, such as injector components or control valves, is difficult to access due to limited optical accessibility, small dimensions and complex geometries as well as high velocities and pressures. Therefore, experimental investigations are mostly performed with upscaled transparent nozzles. For the analysis of cavitation dynamics in orifices and control valves, experiments are carried out with injection into liquid (submerged injection) (Saito and Sato, 2003; Sato and Saito, 2002; Sugimoto and Sato, 2009). Cavitation in injector components and the interaction of cavitation with jet break up was investigated by He et al. (2016); Sou et al. (2014, 2007); Stanley et al. (2011, 2014) with injection into gas. Time-resolved numerical simulations are not limited by the above mentioned constraints and provide three dimensional flow field data to assess the processes in realistic geometries and under real conditions. LES can capture the interaction of cavitation and turbulence, as shown by Egerer et al. (2014). A large number of numerical investigations (Biçer and Sou, 2015; Biçer and Sou, 2016, Edelbauer, 2017; Koukouvinis et al. 2017, Örley et al. 2015 , Trummler et al., 2018) have used the above mentioned reference experiments (Sou et al., 2014, 2007) to validate their modeling approaches and thereby provided deeper insights into the underlying physical processes. Successfully validated models were applied to realistic injector geometries (Koukouvinis et al., 2017; Örley et al., 2017).

Based on the above mentioned studies, two cavitation regimes can be identified. The first regime involves developing cavitation, where cavitation is initiated in the shear layer close to the nozzle inlet and the cavity length reaches about half of the nozzle length (Sou et al., 2007; Stanley et al. 2011). In the separated shear layer at the nozzle inlet, cavitating spanwise vortices form, coalesce, and pair in a rotational motion to larger clouds (Saito and Sato, 2003, Sato and Saito, 2002). In the second regime, known as super cavitation (Chaves et al., 1995), inertia-driven cavitation usually produces a cavity length larger than $80 \%$. Clouds detach from the end of the sheet and collapse close to the nozzle outlet. Stanley et al. (2014) found that transition between these two regimes is characterized by a cavity-length jump and aperiodic shedding. The cavity-length jump can also be seen in other experimental measurements (Saito and Sato, 2003; Sou et al., 2007). In this strongly cavitating regime, the shed clouds can be transported to the nozzle outlet and generate a pressure gradient from the low-pressure vapor region to the outflow, which results in gas being ingested by the nozzle (Örley et al., 2015; Trummler et al., 2018). Further increase of the cavitation length can result in a complete flow detachment from the nozzle wall and the so-called hydraulic flip (Sou et al., 2007; Stanley et al., 2011).

This paper provides a comprehensive analysis of cavitation dynamics in internal nozzle flows with constant cross-section and discharge into ambient gas. We perform compressible implicit LES at two operating points following a reference experiment (Biçer and Sou, 2015; Sou et al., 2014). We analyze cavitation dynamics and the initiating shedding mechanisms. Based on our results, we propose modifications to schematics of the cloud shedding process (Le et al., 1993; Stanley et al. 2014). Moreover, we evaluate the near wall flow field and investigate mechanisms for re-entrant jet 
formation.

We apply a model proposed by Örley et al. (2015), which is an extension of the equilibrium cavitation model of Schnerr et al. (2008) and Schmidt et al. (2014). The thermodynamic model is embedded into a density-based, fully compressible flow solver with an implicit higher-order LES approach for compact stencils (Egerer et al., 2016).

The paper is structured as follows. In section 2 the governing equations, the thermodynamic models and the numerical approach are introduced. Section 3 presents the computational setup. In section 4, we compare our computational results with experimental data, analyze the cavitation dynamics and the shedding mechanisms in detail and relate them to the flow field.

\section{Mathematical and physical model}

\subsection{Governing Equations}

We solve the fully compressible Navier-Stokes equations in conservative form

$$
\partial_{t} \boldsymbol{U}+\nabla \cdot[\boldsymbol{C}(\boldsymbol{U})+\boldsymbol{S}(\boldsymbol{U})]=0 .
$$

The state vector $\boldsymbol{U}=\left[\rho, \rho u_{1}, \rho u_{2}, \rho u_{3}, \rho \xi\right]$ is composed of the conserved variables density $\rho$, momentum $\rho u_{i}$ and a non-condensable gas content $\rho \xi$, where $\xi$ represents the gas mass fraction. Inclusion of non-condensable gas into the model allows for simulating an injection into air as well as the prediction of gas entrainment. Due to the barotropic modeling of the cavitating liquid, combined with an isothermal model of the gas phase, an energy equation is not needed. The convective fluxes $\boldsymbol{C}(\boldsymbol{U})$ across finite-volume cell faces are

$$
\boldsymbol{C}_{i}(\boldsymbol{U})=u_{i} \boldsymbol{U}
$$

and the cell-face stresses $\boldsymbol{S}(\boldsymbol{U})$ are

$$
\boldsymbol{S}_{i}(\boldsymbol{U})=\left[0, \delta_{i 1} p-\tau_{i 1}, \delta_{i 2} p-\tau_{i 2}, \delta_{i 3} p-\tau_{i 3}, 0\right],
$$

where $p$ is the static pressure, $\delta_{i j}$ the Kronecker-Delta and $\boldsymbol{\tau}$ the viscous stress tensor

$$
\tau_{i j}=\mu\left(\partial_{j} u_{i}+\partial_{i} u_{j}+\frac{2}{3} \delta_{i j} \partial_{k} u_{k}\right)
$$

with a dynamic mixture viscosity $\mu$, which depends on the fluid composition in the cell as discussed below.

\subsection{Thermodynamic model}

For the modeling of two-phase flows, homogeneous mixture models (Kubota et al., 1992$)$ describing the multiphase mixture with a compressible effective fluid in a finite volume cell have been established and are widely used for different cavitation regimes, such as re-entrant jet dominated cavitation over a wedge (Gnanaskandan and Mahesh, 2016), condensation shock governed cavitation over a wedge (Budich et al., 2018) and cavitation erosion prediction (Beban et al., 2017, Mihatsch et al., 2015).

Schnerr et al. (2008) formulated a cavitation model based on the assumption that the liquid and gas phases of a cavitating liquid are in thermal and mechanical equilibrium. The thermodynamic equilibrium assumption implies a physically consistent sub-grid model for vapor structures (Schmidt et al., 2014), making such a model particularly suitable for LES. Furthermore, this 
model is parameter-free and able to treat appropriately various cavitation regimes (Egerer et al. 2014). The model has been extensively validated (Beban et al., 2017; Budich et al., 2018; Mihatsch et al. 2015, Schmidt, 2015, Schmidt et al., 2014). Orley et al. (2015) extended the single-fluid cavitation model by a component of non-condensable gas to a multi-component cavitation model and performed LES of the primary break-up phase of cavitating water jets injected into ambient gas.

In this study, we use the multi-component cavitation model of Örley et al. (2015). All components are represented by an effective fluid considering a cell-averaged equilibrium pressure $p$, equilibrium velocity $\boldsymbol{u}$ and Temperature $\vartheta$. Sub-cell phase interfaces are not reconstructed and surface tension is neglected. We consider the components pure liquid, liquid-vapor-mixture, and non-condensable gas, which are denoted by the indices $L, M$, and $G$, respectively. The volume fraction $\beta$ of each component $\Phi=\{L, M, G\}$ within a control volume $V$ is

$$
\beta_{\Phi}=\frac{V_{\Phi}}{V} \quad \text { with } \quad \sum_{\Phi} \beta_{\Phi}=1
$$

and the mass fraction $\xi$ of the mass $m$ of this control volume is

$$
\xi_{\Phi}=\frac{m_{\Phi}}{m} \quad \text { with } \quad \sum_{\Phi} \xi_{\Phi}=1
$$

The density of each component is

$$
\rho_{\Phi}=\frac{m_{\Phi}}{V_{\Phi}}
$$

and the mixture density is

$$
\rho=\sum_{\Phi} \beta_{\Phi} \rho_{\Phi}
$$

Combining pure liquid and liquid-vapor-mixture to $L M$, the mixture density is given by

$$
\rho=\beta_{L M} \rho_{L M}+\beta_{G} \rho_{G}=\left(1-\beta_{G}\right) \rho_{L M}+\beta_{G} \rho_{G} .
$$

In the following, we express $\rho_{L M}$ and $\rho_{G}$ by barotropic relations and combine them to a coupled equation of state (EOS) (Örley et al. 2015. Trummler et al., 2018). The volume fraction of gas $\beta_{G}$ in Eq.99 can be expressed by the transported mass fraction of gas $\xi_{G}$ and the mixture density as

$$
\beta_{G}=\frac{V_{G}}{V}=\frac{m_{G}}{\rho_{G} V}=\frac{m_{G} / m}{\rho_{G} V / m}=\xi_{G} \frac{\rho}{\rho_{G}} .
$$

The equation of state for the pure liquid and the two-phase region is derived from the definition of the isentropic (entropy $s=$ const.) speed of sound $c$

$$
c=\sqrt{\left.\frac{\partial p}{\partial \rho}\right|_{s=\text { const. }}} .
$$

Assuming an approximately constant speed of sound in the liquid region and also in the two-phase region, one can integrate Eq. (11) for each region starting from saturation density $\rho_{\text {sat,liq }}$ to obtain a linearized relation between density and pressure valid in both regions

$$
\rho_{L M}=\rho_{\text {sat }, \text { liq }}+\frac{1}{c^{2}}\left(p-p_{\text {sat }}\right) \text { with } c=\left\{\begin{array}{lll}
c_{L} & \text { if } & p \geq p_{\text {sat }} \\
c_{M} & \text { if } & p<p_{\text {sat }}
\end{array} .\right.
$$


For pure liquid ( $p \geq p_{\text {sat }}$ ) the speed of sound in the liquid $c=c_{L}$ is used and in the two-phase regions we apply the speed of sound of a liquid-vapor-mixture $c=c_{M}$. For water at reference temperature $\vartheta=293.15 \mathrm{~K}$ we set $p_{\text {sat }}=2340 \mathrm{~Pa}, \rho_{\text {sat,liq }}=998.16 \mathrm{~kg} / \mathrm{m}^{3}, c_{L}=1482.35 \mathrm{~m} / \mathrm{s}$ and $c_{M}=1 \mathrm{~m} / \mathrm{s}$ (Örley et al., 2015; Trummler et al., 2018). The vapor content $\alpha$ in a computational cell is calculated from the density of the liquid vapor mixture $\rho_{L M}$ as

$$
\alpha=\frac{\rho_{\text {sat,liq }}-\rho_{L M}}{\rho_{\text {sat,liq }}-\rho_{\text {sat,vap }}}, \quad \text { if } \rho_{L M}<\rho_{\text {sat,liq }}
$$

where $\rho_{\text {sat,vap }}$ denotes the saturation density of the vapor and is $\rho_{\text {sat,vap }}=17.2 \cdot 10^{-3} \mathrm{~kg} / \mathrm{m}^{3}$ at the reference conditions.

The non-condensable gas-phase is modeled as an isothermal ideal gas

$$
\rho_{G}=\frac{p}{\mathrm{R} \vartheta},
$$

with the specific gas constant $\mathrm{R}=287.06 \mathrm{~J} /(\mathrm{kg} \cdot \mathrm{K})$ for air and the reference temperature $\vartheta=$ $293.15 \mathrm{~K}$.

We model the dynamic viscosity of the liquid-vapor mixture $\mu_{L V}$ as proposed by Beattie and Whalley $(1982)$ with

$$
\mu_{L M}=\alpha \cdot \mu_{V}+(1-\alpha)\left(1+\frac{5}{2} \alpha\right) \cdot \mu_{L} .
$$

$\mu_{V}, \mu_{L}$ denote the viscosity of the vapor and liquid, respectively. For a small amount of vapor the viscosity corresponds to vapor phase consisting of small vapor bubbles which behave as solid particles. The viscosity of the mixture fluid considering also the gas component is then approximated by a linear blending with the volume fractions (Trummler et al., 2018)

$$
\mu=\beta_{G} \cdot \mu_{G}+\left(1-\beta_{G}\right) \cdot \mu_{L M} .
$$

The following values for the viscosities are used: $\mu_{L}=1.002 \cdot 10^{-3} \mathrm{~Pa} \cdot \mathrm{s}, \mu_{V}=9.272 \cdot 10^{-6} \mathrm{~Pa} \cdot \mathrm{s}$ and $\mu_{G}=1.837 \cdot 10^{-5} \mathrm{~Pa} \cdot \mathrm{s}$.

\subsection{Numerical approach}

With LES the smallest scales are not resolved on the computational grid and therefore effects of these unresolved sub-grid-scales (SGS) have to be modeled. We employ an implicit LES approach based on the Adaptive Local Deconvolution (ALDM) method by Adams et al. (2004); Hickel et al. (2006, 2014). For implicit LES, the truncation error of the discretization serves as a sub-gridscale model for turbulence. In this work, the implicit LES approach for compact stencils recently proposed by Egerer et al. (2016) is used, which is specially designed for compressible, cavitating flows. Additionally, an extension for the additional gas phase (Trummler et al. 2018) for multiphase application is included.

Shock waves and pseudo phase boundaries are detected by sensor functionals. In the detected non-smooth regions, the velocity components are reconstructed using a third order Total-VariationDiminishing (TVD) slope limiter by Koren (1993), and the thermodynamic quantities $\rho, p$ are reconstructed using the second order minmod slope limiter (Roe, 1986). In smooth regions, a high order central discretization scheme with regularization terms is applied. The extensions introduced for the additional gas phase (Trummler et al. 2018 ) ensure a thermodynamically consistent coupling of the dependent variables pressure, density and gas mass fraction and thus prevent unphysical 


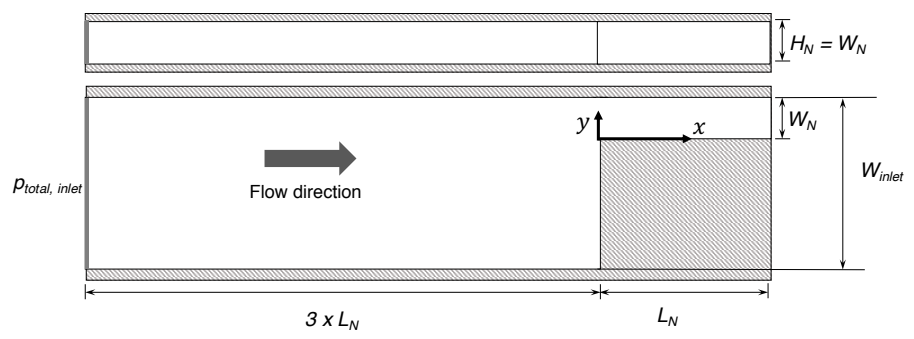

(a)

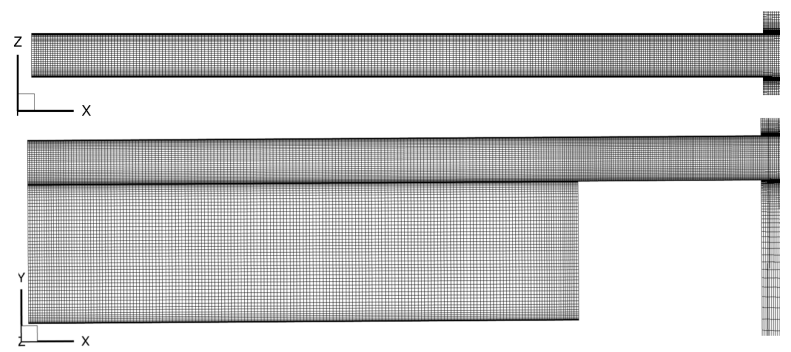

(b)

Figure 1: Numerical setup (a) Sketch of the numerical setup; (b) Slices through the grid on the finest level with every fourth grid line shown. At the nozzle outlet a large domain is added $\left(25 L_{N} \times 75 W_{N} \times 75 W_{N}\right)$ and the outflow region is initialized with gas.

pressure fluctuations. For this purpose, a suitable reconstruction method was implemented to obtain thermodynamically consistent fluxes, especially with regard to the additional flux for the gas phase.

Time integration is performed with an explicit second-order, 4-step low-storage Runge-Kutta method.

\section{Numerical setup}

The numerical setup follows that of an earlier investigation (Trummler et al., 2018). The configuration is adopted from a reference experiment (Biçer and Sou, 2015; Sou et al., 2014) where water is discharged through a transparent step nozzle into ambient air. Different cavitation regimes are obtained by adjusting the inlet pressure and thus the mean velocity in the nozzle. We carry out simulations of two representative operating points, which are listed in Table 1 and explained in more detail in the following section. For both cases, the mean velocity in the nozzle is approximately $\bar{u} \approx 15 \mathrm{~m} / \mathrm{s}$. Fig. 1 depicts the numerical setup. The geometric dimensions are taken from the experiment and have the following values: nozzle length $L_{N}=8 \mathrm{~mm}$, nozzle width and height (quadratic cross-section) $W_{N}=H_{N}=1.94 \mathrm{~mm}$ and width of the inflow region $W_{\text {Inlet }}=8 \mathrm{~mm}$ with the same height as the nozzle $H_{N}$, see Fig. 1. In the following, the nozzle inlet edge is referred to as the origin of an underlying Cartesian coordinate system. For the simulations a large domain extension is added to the nozzle outlet $\left(25 L_{N} \times 75 W_{N} \times 75 W_{N}\right)$ to prevent field boundary conditions from affecting the solution. The length of the inflow region was chosen to be three times the nozzle length and at the inlet a total pressure is prescribed, the values of which are provided 
Table 1: Overview of the operating points investigated.

\begin{tabular}{|cccl|}
\hline $\boldsymbol{\sigma}[-]$ & $\overline{\boldsymbol{u}}[\mathbf{m} / \mathbf{s}]$ & $\boldsymbol{p}_{\text {total,inlet }}\left[10^{5} \mathbf{P a}\right]$ & Cavitation regime \\
\hline 1.19 & 12.8 & 2.37 & developing \\
0.84 & 15.2 & 3.03 & super cavitation \\
\hline
\end{tabular}

in Table 1. The outlet is defined as a pressure outlet with $p_{\text {out }}=1 \cdot 10^{5} \mathrm{~Pa}$, the outlet region is initialized with gas, and the no-slip walls are isothermal.

The simulation domain is discretized on a block-based, structured grid. To reduce computational costs, we employ a grid sequencing strategy. For each operating point, we let the flow field first develop on a coarse grid and then refine the grid over several refinement steps. For a detailed description of this method including a convergence study for a comparable configuration we refer to Örley et al. (2015). Here, we use five different grid levels, where the coarsest grid contains 2.4 million cells and the finest 51.5 million cells. The results presented here are obtained on the finest grid level. On the finest grid, the smallest cell size in wall-normal direction is $2.5 \mu \mathrm{m}$ and the largest cell-size in the nozzle is $30.5 \mu \mathrm{m}$. As can be seen in Fig. 1, the grid is refined near the nozzle walls and around the sharp edge at the nozzle inlet and outlet. Previous investigations of the same configuration (Koukouvinis et al. 2017) and for comparable configurations (Örley et al. 2015) have established grid convergence for the stated resolution.

For wall-bounded turbulent flows, the dimensionless wall normal resolution $y^{+}$is a parameter to evaluate the grid resolution. The analysis of $y^{+}$for this configuration can be found in Trummler et al. (2018): $y^{+} \approx 1$ within the nozzle, i.e. the near-wall cell is within the viscous sublayer.

The high grid resolution and the fully compressible modeling approach result in a time-step of $0.7 \mathrm{~ns}$ on the fine grid with a Courant-Friedrichs-Levy number (CFL) of 1.4. The total simulation time on the finest grid was about $8 \mathrm{~ms}$, for a flow field evolution of $4 \mathrm{~ms}$.

\section{Results}

\subsection{Overview and comparison with experimental data}

The investigated configuration is a step nozzle with quadratic cross-section $\left(W_{N} \times W_{N}\right)$ in which cavitation occurs due to the one-sided constriction and the velocity increase in the detached shear layer. The Reynolds number is

$$
R e=\frac{\rho \bar{u} W_{N}}{\mu},
$$

where $\rho$ denotes the density of the liquid, $\bar{u}$ the mean velocity in the nozzle, $W_{N}$ the nozzle width and $\mu$ the dynamic viscosity. The cavitation number $\sigma$ is defined as

$$
\sigma=\frac{p_{\text {out }}-p_{\text {sat }}}{0.5 \rho \bar{u}^{2}}
$$

where $p_{\text {out }}$ is the pressure at the outlet and $p_{\text {sat }}$ the saturation pressure of the liquid.

We have carried out simulations of two representative operating points at $\sigma=1.19, R e \approx 25,000$ and $\sigma=0.84, R e \approx 30,000$, respectively. Fig. 2 compares the LES results of instantaneous vapor structures with experimental images of the corresponding operating points and shows a good agreement of the cavitation patterns. The operating point $\sigma=1.19$ is in the regime of developing cavitation, where we observe vapor structures in the first half of the nozzle. For $\sigma=0.84$, cavitation 


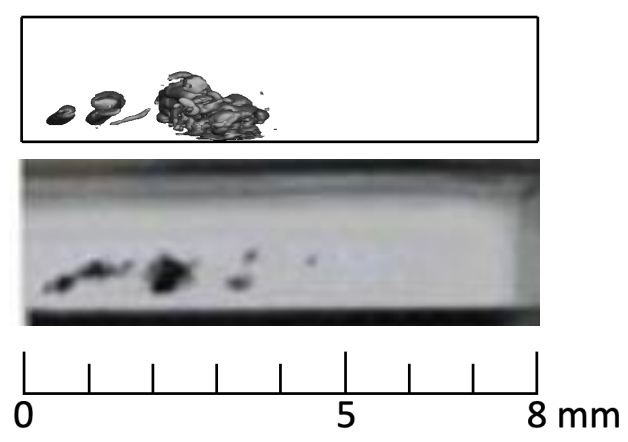

(a)

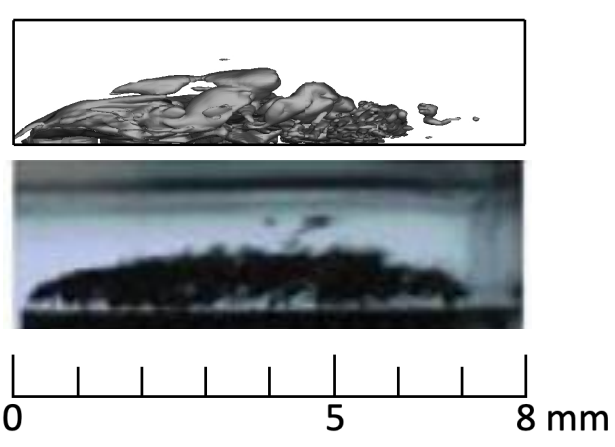

(b)

Figure 2: Comparison of the experimental data (Sou et al. 2014) and the simulation results (Isosurface: vapor $(\alpha=0.1)$ ) (a) $\sigma=1.19$ (b) $\sigma=0.84^{*}\left(\sigma_{\text {Experiment }}=0.82\right)$ (experimental data is reprinted from (Sou et al., 2014)). Flow direction is from left to right.

increases significantly and a vapor sheet extends from the nozzle inlet almost to the outlet. At $\sigma=0.84$ the chosen inlet pressure of the simulation apparently was slightly smaller than in the experiment, resulting in a lower average velocity $\bar{u}(15.2 \mathrm{~m} / \mathrm{s}$ instead of $15.4 \mathrm{~m} / \mathrm{s})$ and thus also in a slightly greater cavitation number than in the experiments.

A validation of the simulation results can be found in Trummler et al. (2018), where the mean streamwise velocity and the velocity fluctuations at different positions are compared with experimental data (Sou et al., 2014) and show good agreement.

\subsection{Cavitation dynamics and shedding mechanisms}

In this section, we analyze the cavitation dynamics and scrutinize the shedding cycles of both operating points. Afterwards, we propose modifications to schematics of the cloud shedding process from the literature for the different shedding mechanisms. Following Stanley et al. (2014), we define the beginning of a cycle by the collapse of the previously shed cloud.

\subsubsection{Developing cavitation and mainly re-entrant jet initiated shedding $(\sigma=1.19)$}

A time series of three shedding cycles depicting instantaneous vapor structures as well as the flow field in a side and a top view is shown in Fig. 4. The corresponding temporal evolution of the vapor content in the nozzle is shown in Fig. 3. The dominant frequency of the integral vapor content based on spectral (Fourier) analysis of the data is $f=1110 \mathrm{~Hz}$ (Trummler et al., 2018), which corresponds to a period of $T=0.9 \mathrm{~ms}$.

At the beginning of each shedding cycle, we observe a single cavity near the nozzle inlet, slightly shifted downstream. The shear layer at the inlet edge contains only a thin vapor sheet. In the middle of each cycle, a vapor cloud is shed and convected downstream, which manifests itself as rolling, cavitating horseshoe vortex, see e.g. fourth column of Fig. 4. Corresponding experimental observations are given in Kubota et al. (1989). These detached, rolling vortices move faster than the main cavity grows, which can be clearly seen from the different trajectories in Fig. 4 (e.g. $t=2.2-2.7 \mathrm{~ms})$. The structures become smaller as they move downstream and their velocity increases. These observations agree with experimental findings by Kubota et al. (1989); Sato and Saito (2002). Furthermore, we observe streamwise cavitating vortices (e.g. $t=0.6 \mathrm{~ms}, t=0.7 \mathrm{~ms}$, 


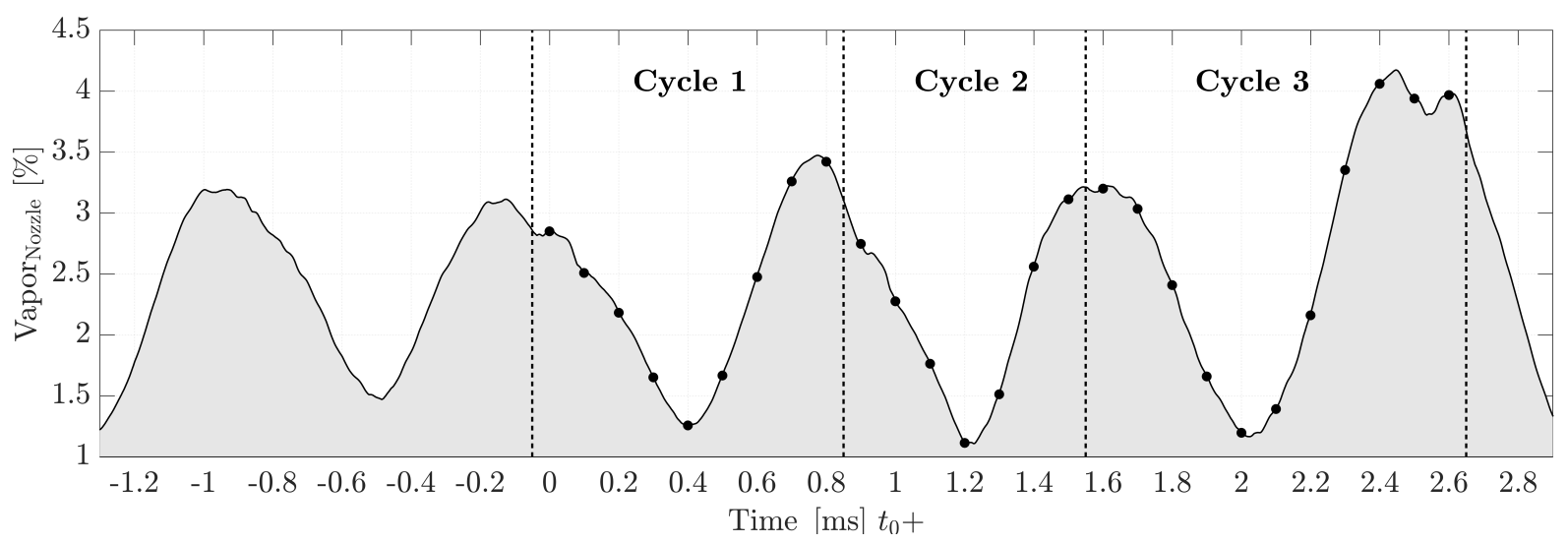

Figure 3: Vapor content over time for $\sigma=1.19$; time steps shown in Fig. 4 are marked with a dot.

$t=2.4 \mathrm{~ms})$. At certain time steps, these connect the main cavity with the detached structure. Saito and Sato (2003) reported similar observations.

After the shedding, a new main cavity forms (black dashed line in Fig. 4), and gains vapor volume by shear layer cavitation. Cavitating spanwise vortices form in the shear layer and later coalesce in a rolling motion (e.g. $t=0.4-0.5 \mathrm{~ms}$ and $t=1.3-1.4 \mathrm{~ms}$ ). Such a pairing of spanwise structures in a rolling motion was also observed in experiments (Saito and Sato, 2003, Sato and Saito, 2002).

The vapor sheet is not a coherent vapor cavity, as often shown schematically (Callenaere et al., 2001; Franc and Michel, 2005, Le et al., 1993), but is a two-phase mixture of vapor bubbles and liquid, which also has been observed in experimental investigations (Kubota et al., 1989; Stanley et al., 2014). Furthermore, in most cases the vapor sheet is not attached to the nozzle wall but separated from the wall by a liquid film with an upstream velocity, as also reported by Stanley et al. (2011, 2014).

The shedding during the first two cycles depicted in Fig. 4is caused by a re-entrant jet, whereas in the last cycle it is due to a condensation shock. During the first two cycles, a thin, coherent, upstream moving liquid film at the end of the sheet can be seen over the first half of the cycle, which agrees with existing schematics of the cloud shedding process (Le et al., 1993; Stanley et al., 2014) depicting a re-entrant jet motion over the first third of a cycle. The jet motion continues upstream and initiates a shedding in the middle of the cycle. An upstream flow underneath the cavity is present during the entire cycle since in the second half of each cycle, the rolling motion of detached vapor structures induces an upstream flow.

During the third cycle, near wall liquid layers are not observed and the vapor reaches down to the wall, i.e. the cavity is attached, which apparently triggers the condensation shock observed during this cycle. Moving upstream the shock increases in height and eventually spans over the entire cavity height (see $t=1.9 \mathrm{~ms}, t=2.0 \mathrm{~ms}$ ).

\subsubsection{Super cavitation and condensation-shock initiated shedding $(\sigma=0.84)$}

Fig. 5 shows a time series covering two shedding cycles. At this operating point, partial gas entrainment occurs. The red isosurface indicates a local gas content of $\geq 10 \%$. Fig. 6 depicts the corresponding temporal evolution of the integral vapor content. The vapor content has increased significantly and does not drop as much as for $\sigma=1.19$. We do not observe a well-defined peri- 


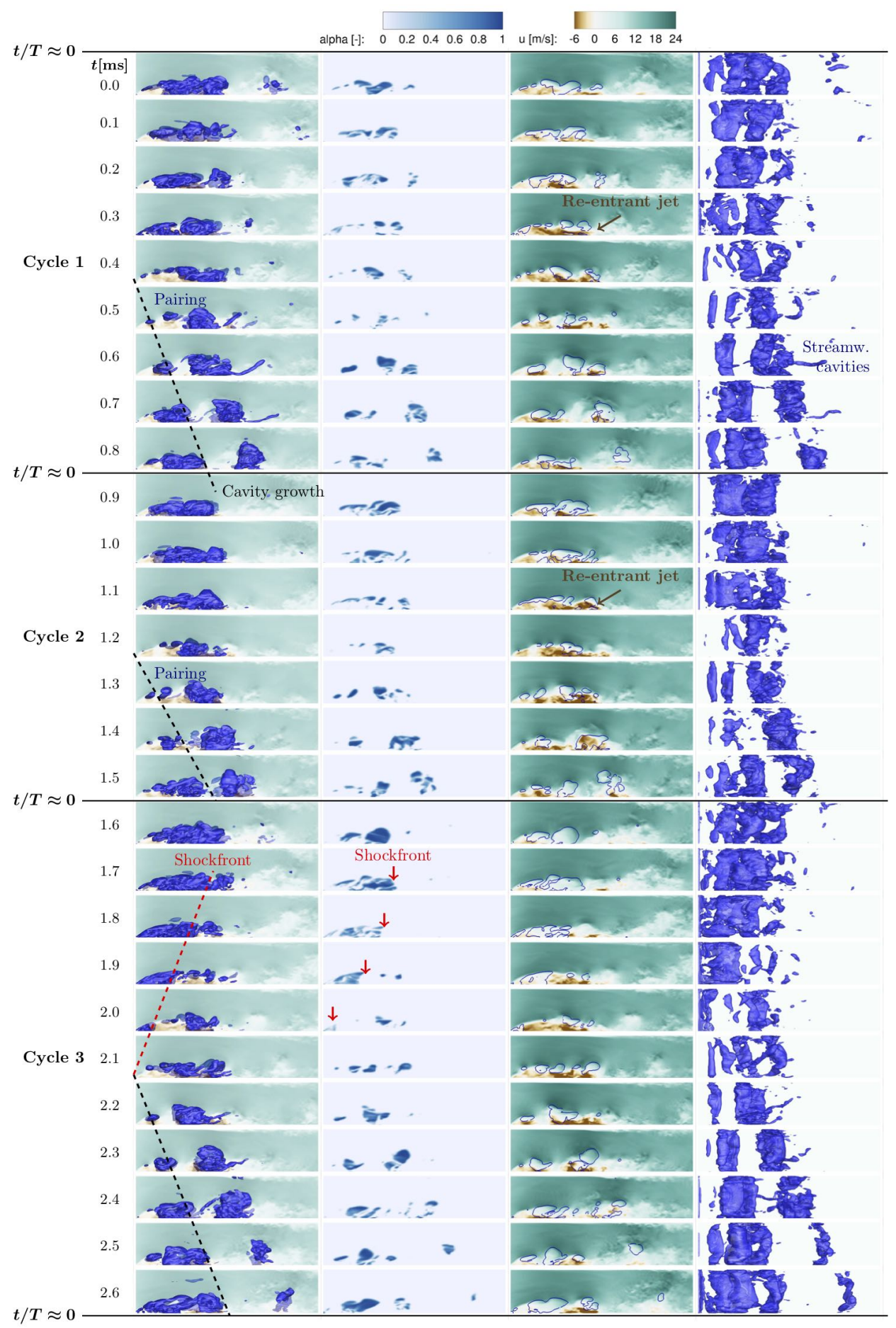

Figure 4: Time series for $\sigma=1.19$, time step $\Delta t=0.1 \mathrm{~ms}$, time increases from top to bottom. First to third column: side view of the midplane flow field; fourth column: top view. First column: streamwise velocity and isosurface vapor; second: vapor; third: streamwise velocity and isoline vapor; fourth: isosurface vapor. Blue isosurface or isoline $10 \%$ vapor, black dashed line indicates the cavity growth and the red dashed one the upstream moving shock front. 


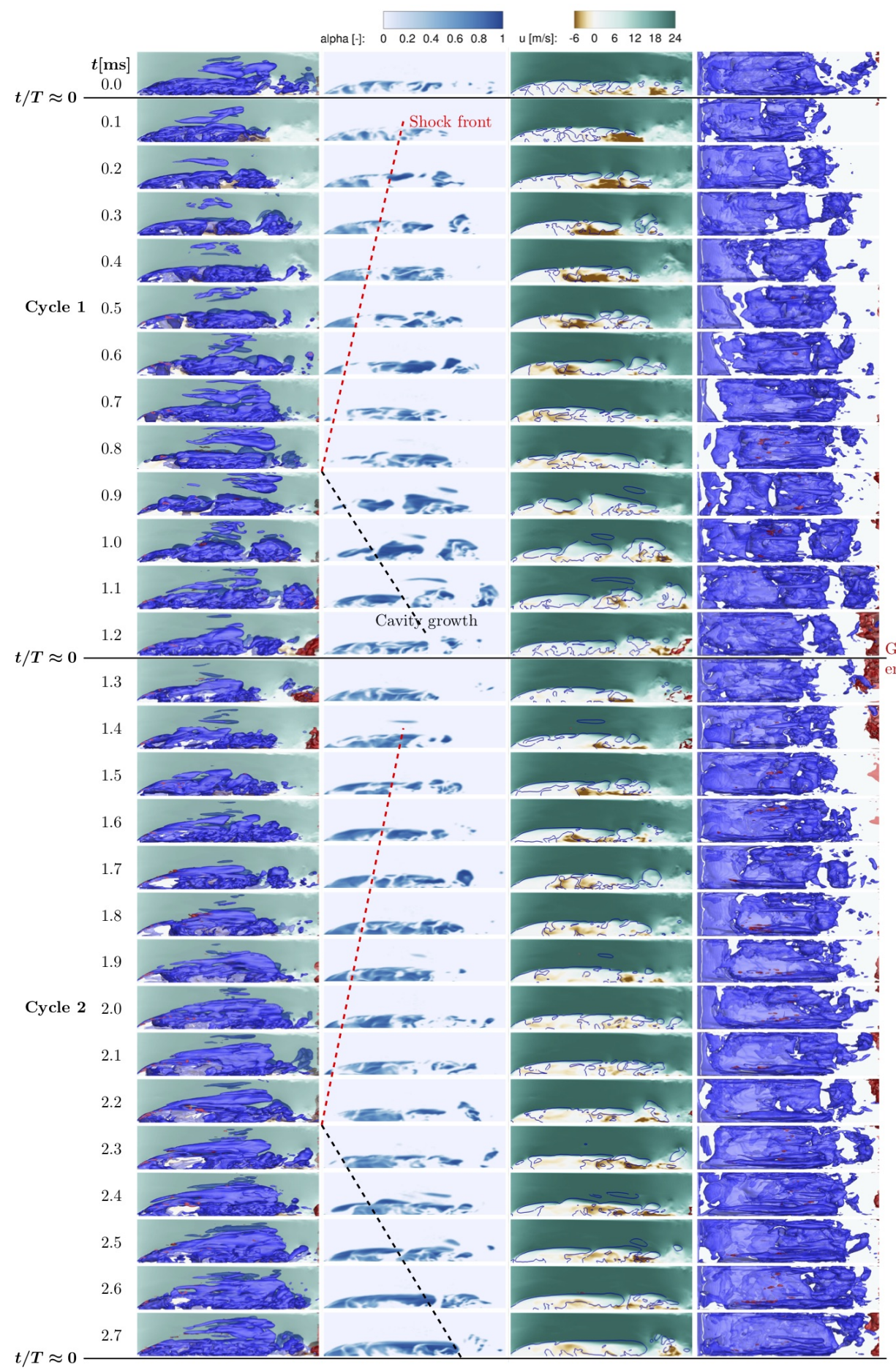

Figure 5: Time series for $\sigma=0.84$, time step $\Delta t=0.1 \mathrm{~ms}$, time increases from top to bottom. First to third column: side view of the midplane flow field; fourth column: top view. First column: streamwise velocity and isosurfaces vapor and gas; second: vapor; third: streamwise velocity and isoline vapor; fourth: isosurfaces vapor and gas. Blue isosurface or isoline $10 \%$ vapor, red isosurface $10 \%$ gas, black dashed line indicates the cavity growth and red dashed one the upstream moving shock front. 


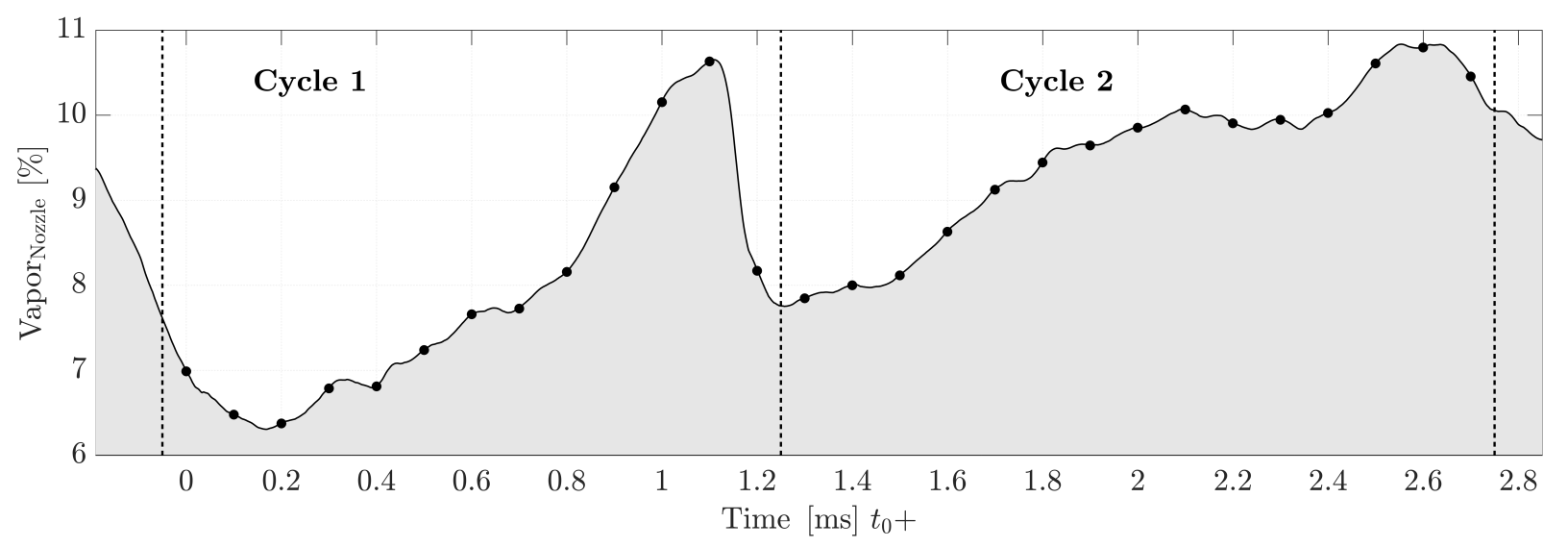

Figure 6: Vapor content over time for $\sigma=0.84$; time steps shown in Fig. 5 are marked with a dot.

odic shedding as for $\sigma=1.19$ and the dominant frequency decreases compared to $\sigma=1.19$, both observations also reported from experimental investigations (Stanley et al., 2011). The dominant frequency found by spectral analysis is $f=750 \mathrm{~Hz}$, which corresponds to $T=1.33 \mathrm{~ms}$. The decreasing shedding frequency with increasing cavitation is consistent with observations by experimental studies (Ganesh, 2015; Ganesh et al., 2016; Stanley et al., 2011).

Streamwise vortices in the center of the nozzle can be observed at some time instants, which also have been observed in experiments (Mauger et al. 2012) and numerical simulations (Egerer et al. 2014); the latter reference discusses the underlying mechanisms leading to the formation of these vortices.

At the beginning of the shedding cycle, a vapor sheet spans from the inlet edge to about $3 / 4$ of the nozzle length. At the end of the sheet, a liquid flow with a comparably high velocity in the upstream direction is visible, similar to the observations and the schematic description by Stanley et al. (2014). During the first half of the shedding cycle, smaller structures at the end of the sheet detach, are convected downstream and then collapse. Due to the detachment of these smaller structures, the cavity length is approximately constant throughout the cycle. The small detached structures are rolling, cavitating spanwise vortices. Occasionally, connecting streamwise vortices are visible, as in $t=0.3 \mathrm{~ms}$ or in $t=1.7 \mathrm{~ms}$. The re-entrant jet formed at the beginning of each cycle later transforms into a condensation shock. While the upstream moving shock leads to the condensation of the front part of the cavity, the rear part is further fed by shear layer cavitation. The complete condensation of the front part causes the detachment of the remaining rear part, see e.g. $t=0.8 \mathrm{~ms}$. This part is then convected further downstream and reshapes into two vapor clouds $(t=0.9-1.2 \mathrm{~ms})$ rotating towards the nozzle outlet. The cloud further downstream reaches the outlet and leads there to gas entrainment into the nozzle $(t=1.2 \mathrm{~ms}$, red isosurfaces). Shortly afterwards $(t=1.3-1.4 \mathrm{~ms})$, the gas is pushed back out. However, a certain amount of gas remains in the nozzle and expands later in the low-pressure vapor region forming small gas-filled structures, see e.g. $t=1.7 \mathrm{~ms}$ and $1.8 \mathrm{~ms}$. The second cloud collapses close to the main cavity $(t=1.2 \mathrm{~ms}$, $t=1.3 \mathrm{~ms}$ ), and after its collapse a thin upstream liquid flow at the end of the cavity can be seen. For the second cycle, we also observe a shedding, though not visible as clearly as in the first cycle depicted, and the volume of the shed cloud is smaller. 
Table 2: Strouhal number $(S t r)$, temporal partitioning $\left(\lambda_{T, \phi}\right)$ and relative velocities $\left(\lambda_{U, \phi}\right)$ with $\phi=\{G, D\}$ where $G$ is growth and $D$ deformation. 'Slope' refers to the lines in the time series (Fig. 4 Fig. 5 ).

\begin{tabular}{|rcccccccccc|}
\hline $\begin{array}{r}\boldsymbol{\sigma} \\
{[-]}\end{array}$ & $\begin{array}{c}\overline{\boldsymbol{u}} \\
{[\mathbf{m} / \mathbf{s}]}\end{array}$ & $\begin{array}{c}\boldsymbol{f} \\
{[\mathbf{H z}]}\end{array}$ & $\begin{array}{c}\boldsymbol{l}_{\mathbf{c a v}} \\
{[\mathbf{m m}]}\end{array}$ & $\begin{array}{c}\boldsymbol{S t r} \\
{[-]}\end{array}$ & $\begin{array}{c}\boldsymbol{\lambda}_{\mathbf{T}, \mathbf{G}} \\
{[-]}\end{array}$ & $\begin{array}{c}\boldsymbol{\lambda}_{\mathbf{u}, \mathbf{G}} \\
{[-]}\end{array}$ & $\begin{array}{c}\boldsymbol{\lambda}_{\mathbf{u}, \mathbf{G}}^{\text {slope }} \\
{[-]}\end{array}$ & $\begin{array}{c}\boldsymbol{\lambda}_{\mathbf{T}, \mathbf{D}} \\
{[-]}\end{array}$ & $\begin{array}{c}\boldsymbol{\lambda}_{\mathbf{u}, \mathbf{D}} \\
{[-]}\end{array}$ & $\begin{array}{c}\boldsymbol{\lambda}_{\mathbf{u}, \mathbf{D}-\mathbf{S F}}^{\text {slope }} \\
{[-]}\end{array}$ \\
\hline 1.19 & 12.8 & 1110 & 3.6 & 0.31 & $1 / 2$ & 0.62 & 0.53 & $1 / 2$ & 0.62 & 0.7 \\
0.84 & 15.2 & 750 & 5.8 & 0.29 & $1 / 3$ & 0.87 & 0.86 & $2 / 3$ & 0.44 & 0.33 \\
\hline
\end{tabular}

\subsubsection{Characterization of the shedding processes}

The shedding at $\sigma=1.19$ is mainly caused by re-entrant jet motion whereas at $\sigma=0.84$ condensation shocks are predominant. The dominance of condensation shocks for the lower cavitation number is in accordance with experimental investigations (Arndt et al., 2001, Jahangir et al., 2018). E.g. Jahangir et al. (2018) determined that for a convergent, divergent nozzle the shedding is shock dominated for $\sigma<0.75$. The comparison to our results indicates that for our nozzle geometry the limits are shifted to higher cavitation numbers, which may result from a stronger blocking effect due to the constant cross-section. Ganesh (2015) found that the formation of condensation shocks is favored at increased void fractions.

The dimensionless Strouhal number for shedding processes is

$$
S t r=f \cdot l_{\text {cav }} / \bar{u}
$$

where $l_{\text {cav }}$ the length of the main cavity, here evaluated in the time series. The values obtained are $S t r \approx 0.3$ (see Table 2 ) and compare well with experimental values for cylindrical orifices with Str $=0.3-0.5$ (Sato and Saito, 2002, Stanley et al., 2011; Sugimoto and Sato, 2009).

As can be seen in the time series (Fig. 4, Fig. 5), shedding cycles can be divided into the upstream motion of a disturbance $(\phi=D)$, which is either a re-entrant jet $(R J)$ or a condensation shock $(C S)$, and the growth phase of the main cavity $(\phi=G)$ with the temporal partitioning of

$$
\lambda_{T, \phi}=t_{\phi} / T \quad \text { and } \quad \sum_{\phi} \lambda_{T, \phi}=1
$$

where $t_{\phi}$ stands for the time the process $\phi=\{D, G\}$ takes. We observe for the re-entrant jet governed cycles at $\sigma=1.19$ a duration of about half a period for each process and thus $\lambda_{T, G}=$ $\lambda_{T, D-R J}=1 / 2$ (see Fig. 4). Our $\lambda_{T, D-R J}$ is slightly higher than reported in the literature as Le et al. (1993) proposed $\lambda_{T, D-R J}=1 / 3$ and Callenaere et al. (2001) found $\lambda_{T, D-R J} \approx 0.40$ and a length of $\lambda_{L, D-R J}=0.75$. For the shock governed regime, the motion of the disturbance is slower and takes about $\lambda_{T, D-S F}=2 / 3$ (see Fig. 5). A dependency of the temporal partitioning on the governing shedding mechanism can also be seen in the time series in Fig. 4 , where the last cycle, with the condensation shock, is significantly longer than the previous ones. In the data provided by Budich et al. (2018) for a condensation shock governed shedding of a cavitating flow over a wedge, the velocity ratios are $\left|u_{G} / u_{D-S F}\right|=1.22$ which implies that $\lambda_{T, G}<\lambda_{T, D-S F}$ and confirms our observation.

The velocity $\left(u_{\phi}=\lambda_{U, \phi} \bar{u}\right)$ of each process is the ratio of the length $l_{\phi}$, which can be expressed as $l_{\phi}=\lambda_{L, \phi} l_{\text {cav }}$, to the time $t_{\phi}$ as

$$
u_{\phi}=\frac{l_{\phi}}{t_{\phi}}=\frac{\lambda_{L, \phi} l_{c a v}}{\lambda_{T, \phi} T},
$$




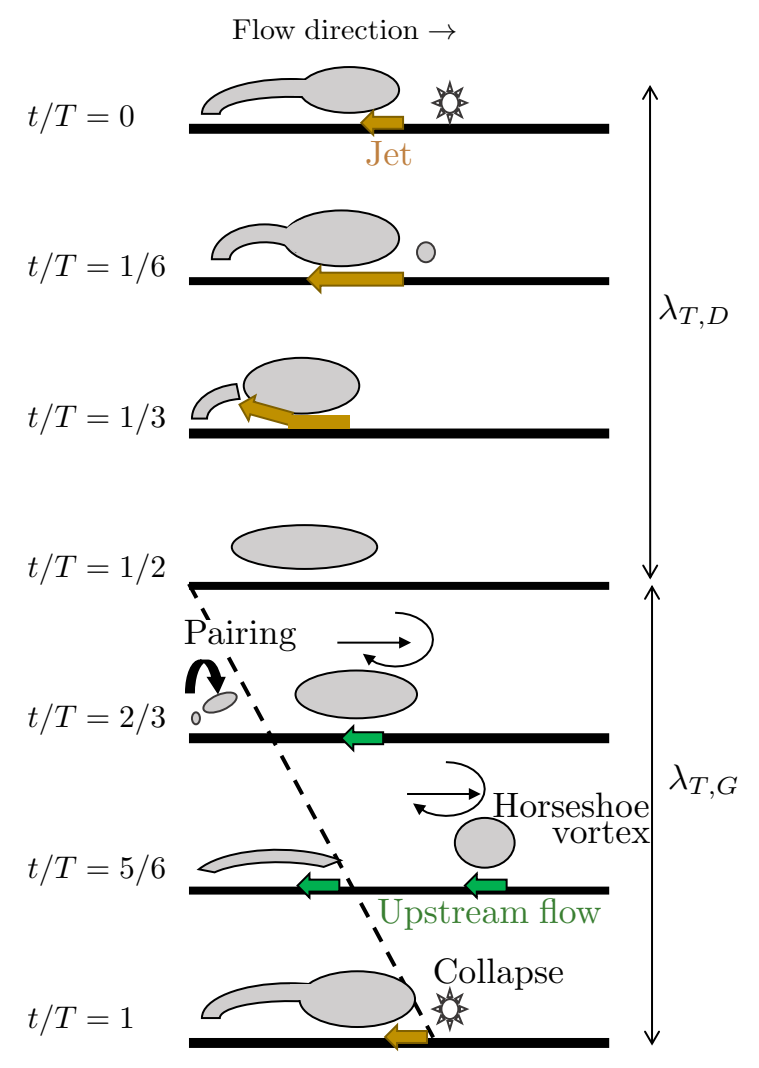

(a)

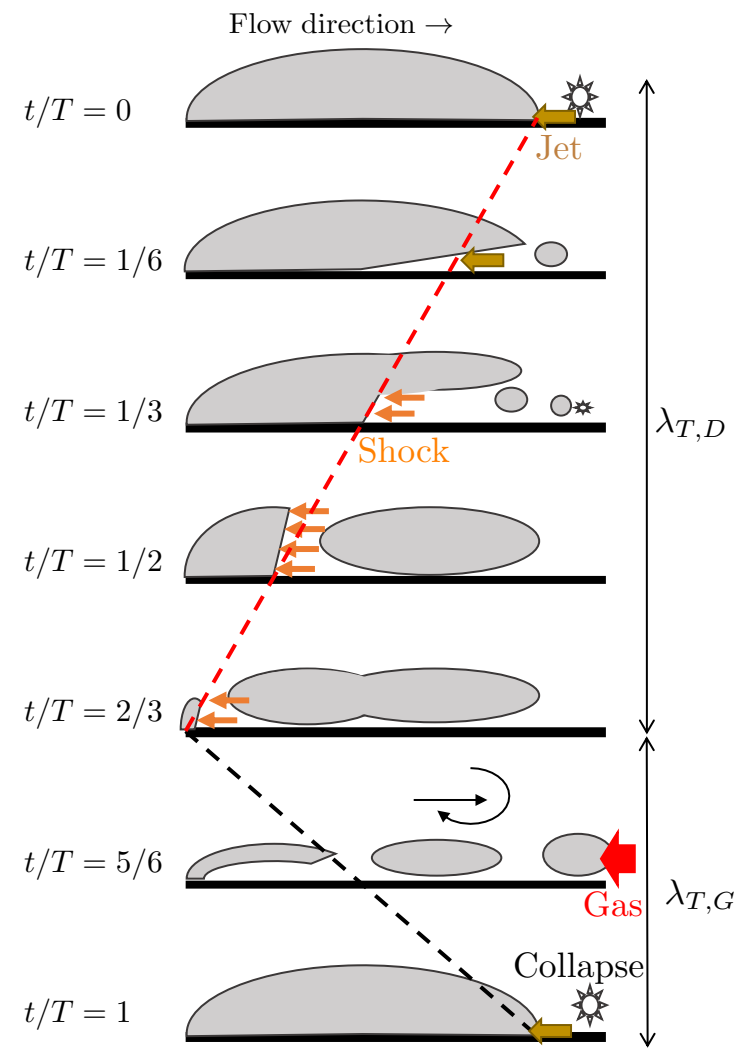

(b)

Figure 7: $\quad$ Schematics of cloud shedding processes adapted and modified from those proposed by Le et al. (1993); Stanley et al. (2014) for re-entrant jet governed cloud shedding. (a) developing cavitation with re-entrant jet initiated shedding (b) super cavitation with condensation shock initiated shedding. Flow direction is from left to right; vapor appears gray.

with $\lambda_{L, \phi} \approx 1$ and Eq. 190 we obtain for the dimensionless velocity

$$
\lambda_{u, \phi} \approx \frac{S t r}{\lambda_{T, \phi}}
$$

Table 2 compares the velocities estimated using Eq. (22), which represent time averaged values, and the ones obtained by the mean slope of the lines tracing the cavity end and the shock front in the time series (Fig. 4, Fig. 5). The agreement of these values shows that Eq. (22) provides a good estimate. Furthermore, the velocities determined and estimated for the disturbance $\lambda_{U, D}, \lambda_{U, D}^{\text {slope }}$ are in accordance with the data from the literature. For re-entrant jets, values of approximately $0.5 \bar{u}$ (e.g. Callenaere et al. (2001); Pham et al. (1999)) are given, which is within the range of our results. Detailed analyses follow in subsection 4.3 . For the shock front velocities values of $35-60 \%$ of the free flow velocity are reported by Ganesh (2015) for cavitating flows over a wedge. For a cavitating nozzle flow, Stanley et al. (2014) observed an upstream moving deformation at the interface of the lower cavity at a velocity of $30-80 \% \bar{u}$ for the range of our cavitation numbers. Both ranges of the shock front velocity agree to our results of $\lambda_{U, D-S F}^{\text {slope }}=0.33-0.7$, see Table 2 . 


\subsubsection{Schematics of the cloud shedding process}

Based on the previous observations we have adapted existing schematics of the cloud shedding process (Le et al., 1993, Stanley et al., 2014) as shown in Fig. 7 and propose modifications depending on the governing shedding mechanism.

For the re-entrant jet initiated shedding at the developing cavitation, an upstream motion of a liquid jet is present during the first half of a cycle. In the middle of the shedding cycle, a vapor cloud is shed and convected downstream as a rolling horse-shoe vortex. The growth of the new main cavity is due to shear layer cavitation and paring and coalescence of the formed spanwise vortices. An upstream, liquid, near-wall flow is present throughout the cycle - first due to the re-entrant jet motion and later due to the rolling motion of the detached structures.

In case of a shock initiated shedding the vapor sheet is attached to the nozzle wall. At the beginning of a cycle, a re-entrant jet forms at the end of the sheet and moves upstream transforming into a condensation shock. Here the temporal partitioning into the convection of the disturbance is longer than for the re-entrant jet initiated shedding, as also discussed above. The condensation of the upstream part leads to the detachment of the rear part. In this cavitation regime, detached vapor clouds reaching the nozzle outlet can lead to gas entrainment. In this study, the gas entrainment affects only the flow field directly at the nozzle outlet, and we did not observe a direct impact on cavitation dynamics and shedding.

\subsection{Flow field}

In this subsection special emphasis is placed on the near-wall upstream flow and the investigation of the underlying mechanisms leading to the re-entrant jet formation.

\subsubsection{Instantaneous flow field and vorticity}

The instantaneous flow field and the vorticity distribution on the midplane for one time step of each operating point are shown in Fig. 8. In addition, data extracted at the nozzle inlet and across a detached vapor structure are plotted at the bottom. In Fig. 8, the flow detaches at the nozzle inlet and the highest velocity occurs in the detached shear layer, where the liquid starts to cavitate. The velocity above the cavity $\left(u_{\text {cav }}\right)$ can be approximated by Bernoulli's equation applied to a streamline from the inlet to the cavity

$$
u_{\text {cav }}=\sqrt{2 \cdot\left(p_{\text {total }, \text { inlet }}-p_{\text {sat }}\right) / \rho} \text {. }
$$

$u_{\text {cav }}$ is $21.66 \mathrm{~m} / \mathrm{s}$ and $24.54 \mathrm{~m} / \mathrm{s}$ for $\sigma=1.19$ and $\sigma=0.84$ respectively, which closely matches the velocities at the cavity interface in Fig. 8. At $\sigma=1.19$ the velocity on the upper side of the vapor structures increases locally due to displacement, whereas at $\sigma=0.84$ the velocity above the detached shear layer is approximately constant.

The flow around the cavity induces a negative circulation $\Gamma_{\text {cav }}$ in $z$ direction. Circulation $\Gamma$ is the line integral of the velocity $\underline{u}$ along a closed line $\underline{s}$

$$
\Gamma=\oint_{\partial s} \underline{u} d \underline{s}
$$

or, due to Stokes' theorem, the integral of the vorticity $\underline{\omega}=\nabla \times \underline{u}$ over the enclosed area $A$ with the normal vector $\underline{n}$ as

$$
\Gamma=\int_{A}(\nabla \times \underline{u}) \cdot \underline{n} d A=\int_{A} \underline{\omega} \cdot \underline{n} d A .
$$




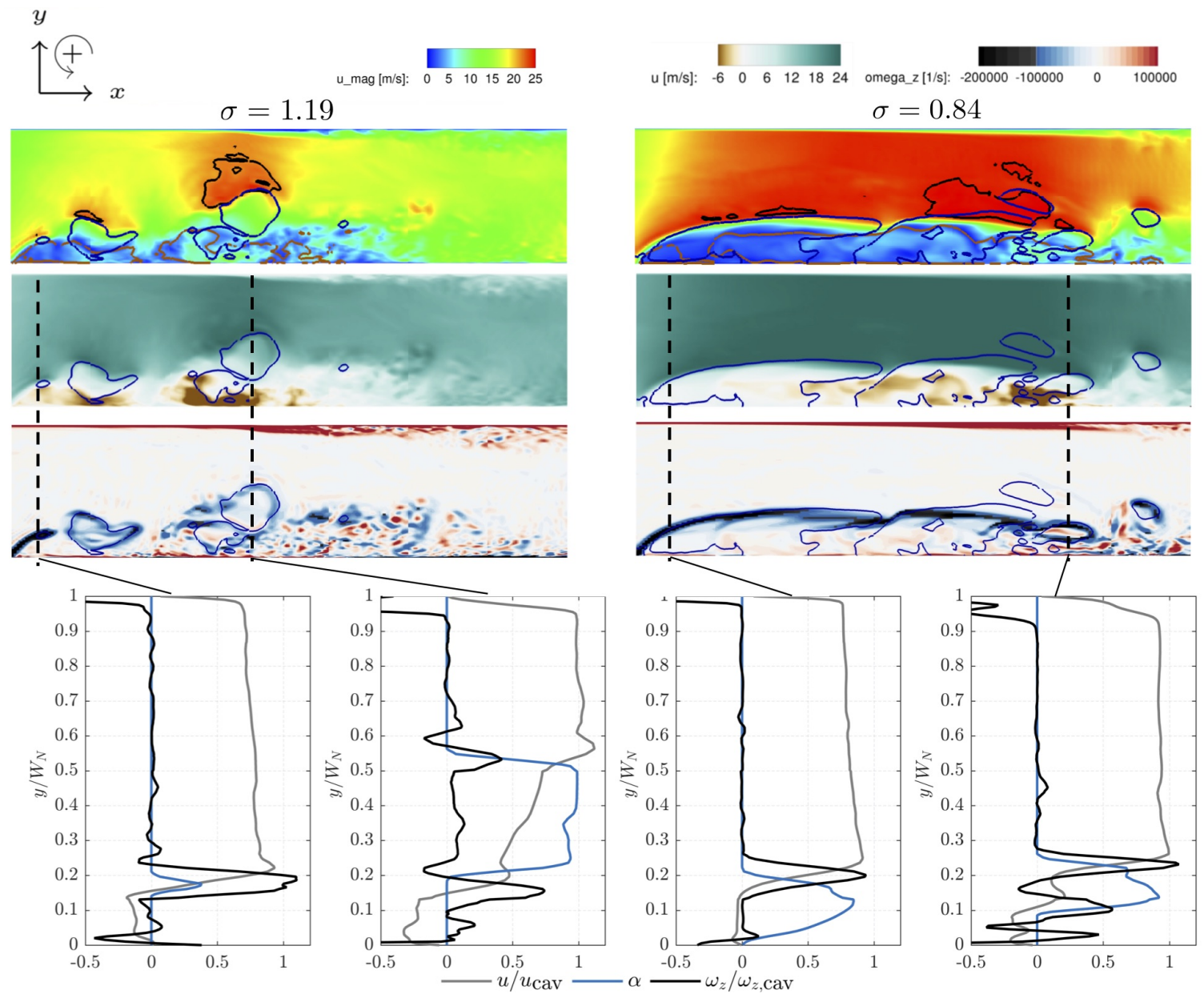

Figure 8: Flow field and vorticity on the midplane $(x-y)$. Left for $\sigma=1.19$ at $t=2.3 \mathrm{~ms}$, right for $\sigma=0.84$ at $t=2.5 \mathrm{~ms}$. First row: velocity magnitude and isolines $u_{\text {cav }}$ (black), $u=0 \mathrm{~m} / \mathrm{s}$ (brown) and $\alpha=10 \%$ (blue); second: streamwise velocity and isoline $\alpha=10 \%$ (blue); third: vorticity and isoline $\alpha=10 \%$ (blue); at the bottom: extracted data at the marked positions. 
$\Gamma_{\text {cav }}$ can be estimated by using Eq. (24) with an integration path along the cavity interface, see e.g. Le et al. (1993), as

$$
\left|\Gamma_{\text {cav }}\right| \approx u_{\text {cav }} \cdot l_{\text {cav }} .
$$

Fig. 8 shows that at $\sigma=0.84$ the vorticity $\omega_{z}$ for this circulation is concentrated in a thin layer at the boundary of the cavity. Thus, $\Gamma_{\text {cav }}$ can be approximated with Eq. (25) by the vorticity in this layer $\omega_{z \text {,cav }}$ and the area, where it is present $\left(l_{\text {cav }} \cdot h_{\text {vort }}\right)$, as

$$
\Gamma_{\mathrm{cav}} \approx \omega_{z, \mathrm{cav}} \cdot l_{\mathrm{cav}} \cdot h_{\mathrm{vort}}
$$

and with Eq. 26) we obtain

$$
\omega_{z, \mathrm{cav}} \approx-u_{\mathrm{cav}} / h_{\mathrm{vort}} .
$$

We estimate $h_{\text {vort }} \approx 0.1 \mathrm{~mm}$ and the derived values for $\omega_{z \text {,cav }}$ correspond at both operating points to the vorticity at the shear layer at the nozzle inlet, see Fig. 8 ,

At $\sigma=1.19$, the highly vortical shear layer transitions into spanwise vortices, which are surrounded by vorticity of reduced magnitude. At both operating points, the shed vapor structures downstream are framed by layers of negative vorticity of about $0.5-1 \omega_{z \text {,cav }}$. Within these structures, the magnitude of the vorticity is either significantly decreased $(\sigma=1.19)$ or decreases radially $(\sigma=0.84)$, indicating that vapor structures do not rotate like a rigid body. Note that at $\sigma=1.19$ the data is extracted across a vapor structure which is pairing in a rolling motion with another one.

Underneath the detached vapor structures at $\sigma=1.19$ and at the end of the attached sheet of $\sigma=0.84$, there is a strongly pronounced upstream flow of about $0.3 u_{\text {cav }}$ that correlates to a high negative vorticity at these locations.

Additionally, we want to emphasize here the strong interaction of vortical structures and cavitation. Cavitation often occurs in the low pressure cores of these structures. The time series in Fig. 4 and 5 show streamwise and spanwise vortical structures. Vortex cavitation is assessed in detail in e.g. Arndt (2002); Cheng et al. (2020); Ji et al. (2015, 2014).

\subsubsection{Near-wall upstream flow}

We analyze the near-wall upstream flow using $x$-t diagrams in which the flow field on the midplane close to the wall is extracted and arranged with increasing time, see Fig. 9. Furthermore, the pressure field at the end of the cavitation zone is depicted in the second column in Fig. 9. The upstream velocity at $l / l_{\text {cav }}=\{0.15,0.5,0.85\}$ is plotted over time in Fig. 10 .

In the $x$ - $t$ diagram of $\sigma=1.19$ the formation of a re-entrant jet at the beginning of each cycle and its upstream movement can be seen. The highest velocity peaks occur at the end of the sheet at about $2 / 3$ of each cycle, see also Fig. 10, and are apparently induced by the rolling motion of the detached structures, see Fig. 4 and subsection 4.3.1. Additionally, this upstream flow can be amplified by the pressure peak induced by the collapse of smaller vapor structures at the end of the sheet, see middle of second cycle. Furthermore, the extracted velocities (Fig. 10) illustrate that the velocity magnitude tends to decrease in upstream direction.

For $\sigma=0.84$, the $x$ - $t$ diagram (Fig. 9) and the extracted velocity (Fig. 10) show a clear and unique peak at the end of the cavitation zone at the beginning of the two cycles, which corresponds to the re-entrant jet formed there. A comparison with the pressure field (left column of Fig. 9) reveals that negative velocity peaks correlate with pressure peaks induced by the collapse of the shed cloud. The fluid with peak velocity moves upstream and slows down. In the $x$ - $t$ diagram it can be seen that further upstream the jet and the upstream flow are not purely liquid anymore and the jet 


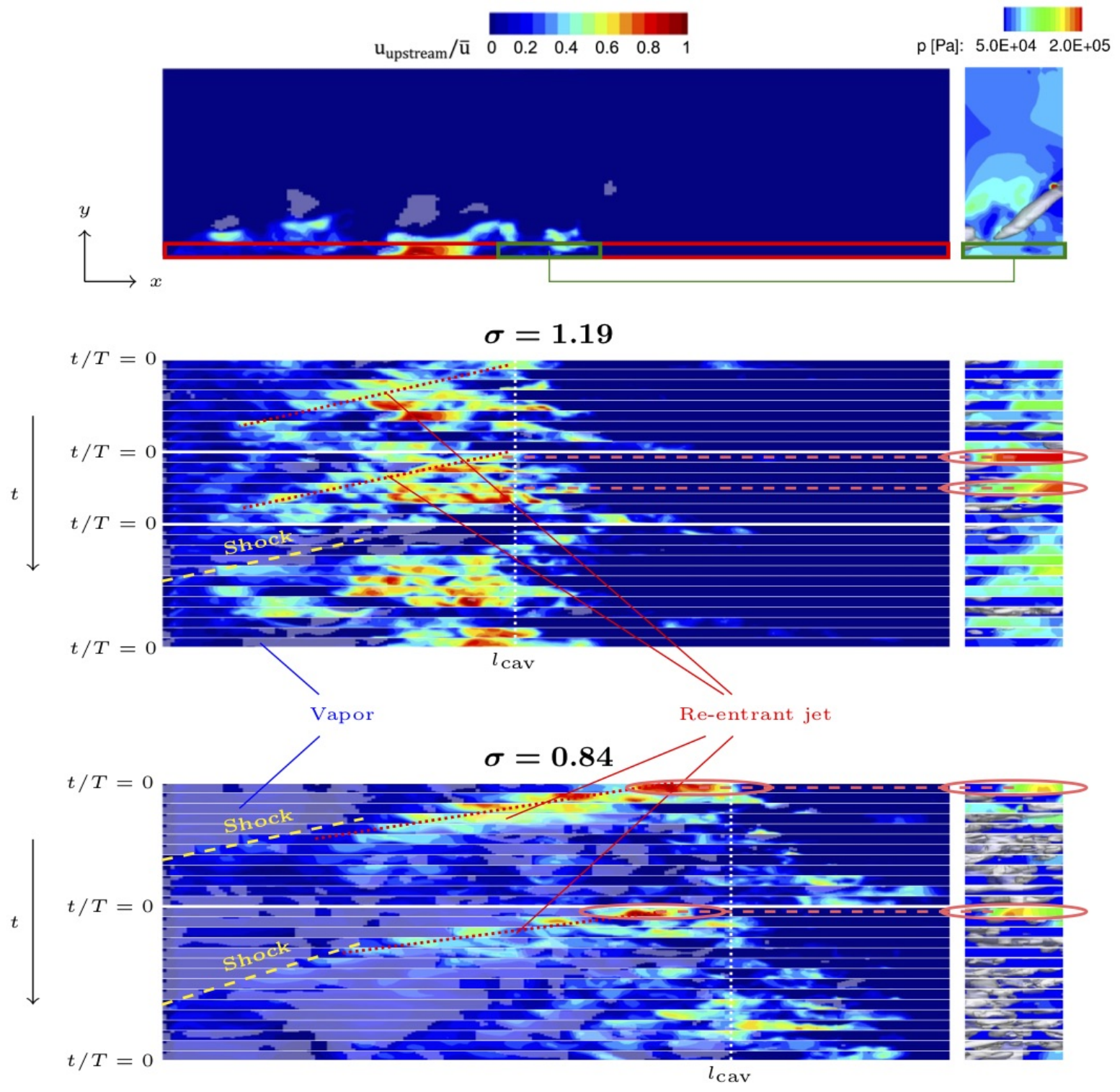

Figure 9: Time series $(x-t)$ of streamwise velocity $u$ (left) and pressure field $p$ (right) close to the wall $(h=0.1 \mathrm{~mm})$. Vapor regions $(\alpha=0.1)$ are indicated as bright regions (left) and as white isosurface(right). The top panel illustrates the investigated regions. Red dotted lines indicate the re-entrant jet motion and yellow dashed lines highlight the shock front. Pressure peaks downstream of the cavity are correlated to the velocity field and are marked in orange. 

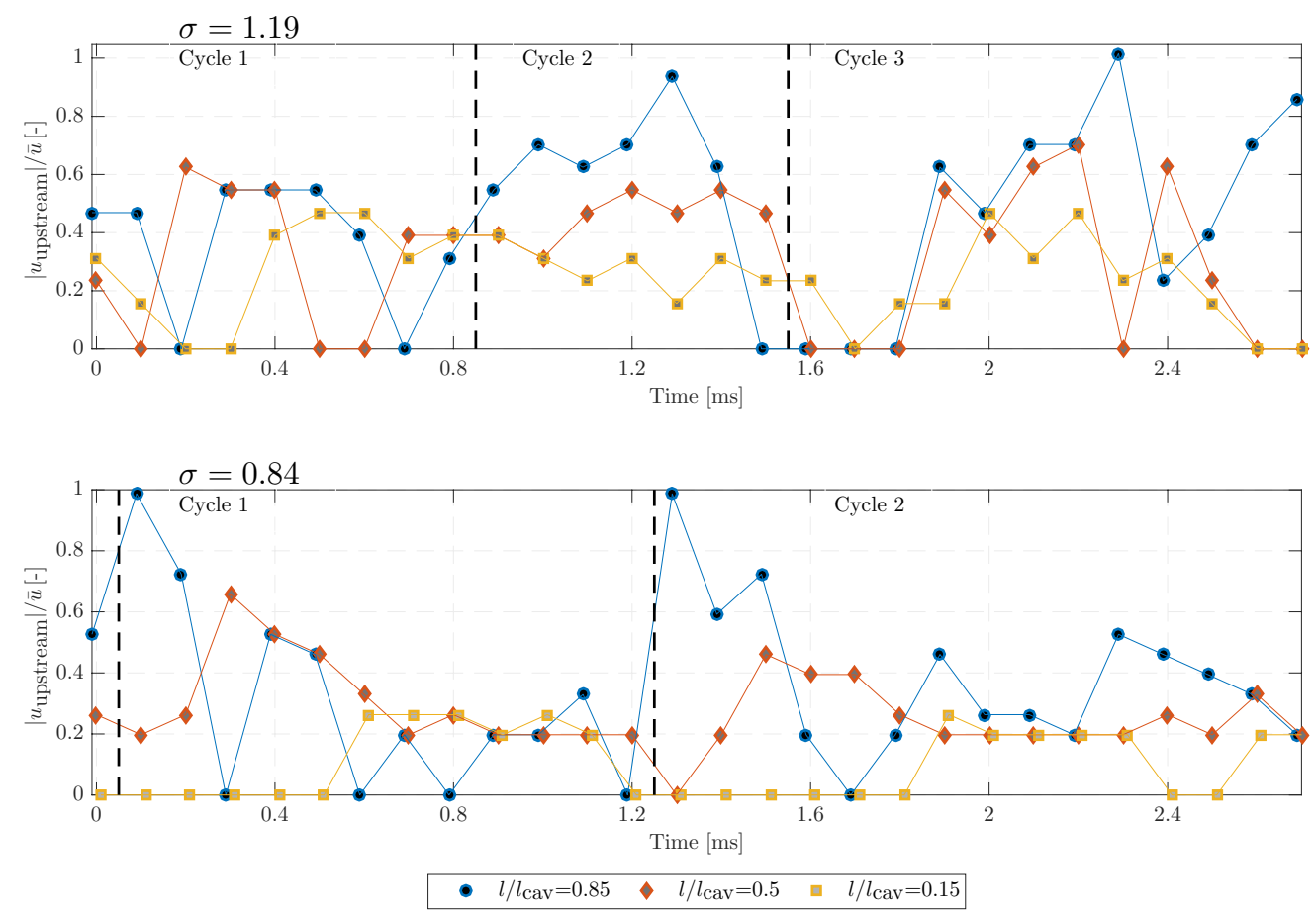

Figure 10: Near-wall upstream velocity normalized by mean nozzle velocity $\left|u_{\text {upstream }}\right| / \bar{u}$ evaluated at different positions as a function of time.

also extends into the vapor region. While moving upstream the jet transforms into a condensation shock, which is clearly visible by the upstream moving vapor front in the $x$ - $t$ diagram. Close to the nozzle inlet (see $0.15 l_{\text {cav }}$ in Fig. 10), there is only an upstream flow after the condensation shock has passed. In the second half of the cycles, the velocity magnitudes at all three positions are about the same $(20-30 \%$ of $\bar{u})$, which agrees with the measured data of Stanley et al. (2014). We point out that Stanley et al. (2014) reported an upstream moving deformation, which we believe is related to a condensation shock.

The re-entrant jet corresponds, in our notation of the shedding cycles, to the upstream flow at the beginning of the cycle. The determined re-entrant jet velocity magnitudes have peaks in the range of the mean velocity $\bar{u}$ at the end of the cavity and decrease in magnitude in upstream direction, which is in good agreement with experimental observations (Pham et al., 1999; Sakoda et al. 2001). At $\sigma=1.19$, the re-entrant jet velocities are slightly lower and match well with the re-entrant jet velocity of half the mean velocity determined by Callenaere et al. (2001).

So far, the driving mechanism behind the re-entrant jet has not yet been completely clarified. In our simulations we observe for $\sigma=0.84$ a clear correlation between the collapse of the shed cloud and a more pronounced upstream flow, which was proposed in the literature by e.g. Leroux et al. (2004, 2005). However, at $\sigma=1.19$ we observe this correlation only once, which matches the finding by Coutier-Delgosha et al. (2007) that this relation is flow condition dependent. Moreover, we found that at $\sigma=1.19$ the highest magnitude of the upstream flow is induced by the rolling motion of detached vapor structures and not associated to the re-entrant jet motion. 


\section{Conclusion}

Cloud cavitation is a common yet challenging phenomenon that occurs in external and in internal cavitating flows. Although orifices and nozzles with constant cross-section play an important role in technical applications, detailed investigations of cavitation dynamics and shedding mechanisms have been far less performed than for external flows or for flows in convergent-divergent geometries.

In this paper, cavitation dynamics and shedding mechanisms of cavitating nozzle flows were investigated using wall-resolved LES results. The three-dimensional flow field data of several shedding cycles provide a deep insight into the physical processes of the cloud cavitation mechanisms in nozzle flows and allow detailed analyses of the flow field. Our results compare well with the reference experiment and reported observations for cavitation dynamics.

In our simulations, the shedding for inertia-driven super cavitation $(\sigma=0.84)$ is initiated by condensation shocks, whereas for developing cavitation $(\sigma=1.19)$ it is primarily initiated by reentrant jets. The occurrence of condensation shocks at lower cavitation numbers is consistent with experimental findings. Our investigation reveals for the first time condensation shocks in cavitating nozzle flows with constant cross-section. Based on our observations, we have analyzed the shedding in detail and then adapted and extended the schematics of the cloud shedding process from the literature for the different mechanisms.

At both operating points, a re-entrant jet forms at the beginning of the cycle and in case of shock-initiated shedding the jet transforms further upstream into a shock. Our results confirm the existing theory that the re-entrant jet formation is related to the pressure peak induced by the collapse of the detached cloud. The determined re-entrant jet velocities are in the order of the mean nozzle velocity and decrease in upstream direction. For developing cavitation a pronounced upstream flow is present during the entire cycle, which first can be characterized as re-entrant jet motion and later as an upstream flow induced by the rolling motion of the detached vapor structures, which lead to the highest upstream velocities.

The analysis performed contributes to an improved understanding of cavitation dynamics in nozzles and has demonstrated the occurrence of condensation shocks there. Furthermore, this paper provides detailed data on the shedding processes and the near-wall upstream flow and therewith complements the existing literature with data for cavitating flows in a rectangular step nozzle with injection into gas.

\section{Acknowledgments}

The authors acknowledge the Gauss for Supercomputing e.V. for granting computational resources on the GCS Supercomputer SuperMUC at the Leibniz Supercomputing Centre (LRZ, www.lrz.de). Nikolaus A. Adams acknowledges support through the ERC Advanced Grant NANOSHOCK (2015).

\section{References}

Adams, N., Hickel, S., Franz, S., 2004. Implicit subgrid-scale modeling by adaptive deconvolution. Journal of Computational Physics 200 (2), 412-431.

Arndt, R. E., 2002. Cavitation in vortical flows. Annual review of fluid mechanics 34 (1), 143-175. 
Arndt, R. E. A., Song, C. C. S., Kjeldsen, M., Keller, A., 2001. Instability of partial cavitation: A numerical/experimental approach. In: Twenty-Third Symposium on Naval Hydrodynamics Office of Naval Research Bassin d'Essais des Carenes National Research Council. pp. 599-615.

Asi, O., 2006. Failure of a diesel engine injector nozzle by cavitation damage. Engineering Failure Analysis 13 (7), 1126-1133.

Beattie, D. R. H., Whalley, P. B., 1982. A simple two-phase frictional pressure drop calculation method. International Journal of Multiphase Flow 8 (1), 83-87.

Beban, B., Schmidt, S., Adams, N., 2017. Numerical study of submerged cavitating throttle flows. Atomization and Sprays 27 (8), 723-739.

Biçer, B., Sou, A., 2015. Numerical models for simulation of cavitation in diesel injector nozzles. Atomization and Sprays 25 (12), 1063-1080.

Biçer, B., Sou, A., 2016. Application of the improved cavitation model to turbulent cavitating flow in fuel injector nozzle. Applied Mathematical Modelling 40 (7-8), 4712-4726.

Budich, B., Schmidt, S. J., Adams, N. A., 2018. Numerical simulation and analysis of condensation shocks in cavitating flow. Journal of Fluid Mechanics 838, 759-813.

Callenaere, M., Franc, J.-P., Michel, J.-M., Riondet, M., 2001. The cavitation instability induced by the development of a re-entrant jet. Journal of Fluid Mechanics 444, 223-256.

Chaves, H., Knapp, M., Kubitzek, A., Obermeier, F., Schneider, T., 1995. Experimental study of cavitation in the nozzle hole of diesel injectors using transparent nozzles. SAE International, $645-657$.

Cheng, H., Bai, X., Long, X., Ji, B., Peng, X., Farhat, M., 2020. Large eddy simulation of the tip-leakage cavitating flow with an insight on how cavitation influences vorticity and turbulence. Applied Mathematical Modelling 77, 788-809.

Coutier-Delgosha, O., Stutz, B., Vabre, A., Legoupil, S., 2007. Analysis of cavitating flow structure by experimental and numerical investigations. Journal of Fluid Mechanics 578, 171-222.

De Lange, D. F., De Bruin, G. J., 1998. Sheet Cavitation and Cloud Cavitation, Re-Entrant Jet and Three-Dimensionality. In: Biesheuvel, A., van Heijst, G. F. (Eds.), Fascination of Fluid Dynamics. Springer, pp. 91-114.

Edelbauer, W., 2017. Numerical simulation of cavitating injector flow and liquid spray break-up by combination of eulerian-eulerian and volume-of-fluid methods. Computers \& Fluids 144, 19-33.

Egerer, C. P., Hickel, S., Schmidt, S. J., Adams, N. A., 2014. Large-eddy simulation of turbulent cavitating flow in a micro channel. Physics of Fluids 26 (8), 085102.

Egerer, C. P., Schmidt, S. J., Hickel, S., Adams, N. A., 2016. Efficient implicit les method for the simulation of turbulent cavitating flows. Journal of Computational Physics 316 (C), 453-469.

Franc, J.-P., Michel, J.-M., 2005. Fundamentals of cavitation. Dordrecht: Springer science \& Business media. 
Furness, R., Hutton, S., 1975. Experimental and theoretical studies of two-dimensional fixed-type cavities. Journal of Fluids Engineering 97 (4), 515-521.

Ganesh, H., 2015. Bubbly shock propagation as a cause of sheet to cloud transition of partial cavitation and stationary cavitation bubbles forming on a delta wing vortex. Ph.D. thesis.

Ganesh, H., Mäkiharju, S. A., Ceccio, S. L., 2016. Bubbly shock propagation as a mechanism for sheet-to-cloud transition of partial cavities. Journal of Fluid Mechanics 802, 37-78.

Gnanaskandan, A., Mahesh, K., 2016. Large eddy simulation of the transition from sheet to cloud cavitation over a wedge. International Journal of Multiphase Flow 83, 86-102.

Gopalan, S., Katz, J., 2000. Flow structure and modeling issues in the closure region of attached cavitation. Physics of Fluids 12 (4), 895-911.

Hayashi, S., Sato, K., 2014. Unsteady Behavior of Cavitating Waterjet in an Axisymmetric Convergent-Divergent Nozzle: High Speed Observation and Image Analysis Based on Frame Difference Method. Journal of Flow Control, Measurement \& Visualization 02 (03), 94-104.

He, Z., Chen, Y., Leng, X., Wang, Q., Guo, G., 2016. Experimental visualization and LES investigations on cloud cavitation shedding in a rectangular nozzle orifice. International Communications in Heat and Mass Transfer 76 (C), 108-116.

Hickel, S., Adams, N. A., Domaradzki, J. A., 2006. An adaptive local deconvolution method for implicit les. Journal of Computational Physics 213 (1), 413-436.

Hickel, S., Egerer, C. P., Larsson, J., 2014. Subgrid-scale modeling for implicit large eddy simulation of compressible flows and shock-turbulence interaction. Physics of Fluids 26 (10), 106101.

Jahangir, S., Hogendoorn, W., Poelma, C., 2018. Dynamics of partial cavitation in an axisymmetric converging-diverging nozzle. International Journal of Multiphase Flow 106, 34-45.

Jakobsen, J., 1964. On the mechanism of head breakdown in cavitating inducers. Journal of Basic Engineering 86 (2), 291-305.

Ji, B., Luo, X., Arndt, R. E., Peng, X., Wu, Y., 2015. Large eddy simulation and theoretical investigations of the transient cavitating vortical flow structure around a naca66 hydrofoil. International Journal of Multiphase Flow 68, 121-134.

Ji, B., Luo, X., Arndt, R. E., Wu, Y., 2014. Numerical simulation of three dimensional cavitation shedding dynamics with special emphasis on cavitation-vortex interaction. Ocean Engineering $87,64-77$.

Kawanami, Y., Kato, H., Yamaguchi, H., Tanimura, M., Tagaya, Y., 1997. Mechanism and control of cloud cavitation. Journal of Fluids Engineering 119 (4), 788-794.

Koren, B., 1993. A robust upwind discretization method for advection, diffusion and source terms. Centrum voor Wiskunde en Informatica Amsterdam.

Koukouvinis, P., Naseri, H., Gavaises, M., 2017. Performance of turbulence and cavitation models in prediction of incipient and developed cavitation. International Journal of Engine Research 18 (4), 333-350. 
Kubota, A., Kato, H., Yamaguchi, H., 1992. A new modelling of cavitating flows: a numerical study of unsteady cavitation on a hydrofoil section. Journal of Fluid Mechanics 240, 59-96.

Kubota, A., Kato, H., Yamaguchi, H., Maeda, M., 1989. Unsteady structure measurement of cloud cavitation on a foil section using conditional sampling technique. Journal of Fluids Engineering 111 (2), 204-210.

Laberteaux, K., Ceccio, S., 2001. Partial cavity flows. part 1. cavities forming on models without spanwise variation. Journal of Fluid Mechanics 431, 1-41.

Le, Q., Franc, J.-P., Michel, J.-M., 1993. Partial cavities: global behavior and mean pressure distribution. Journal of Fluids Engineering 115 (2), 243-248.

Leroux, J. B., Astolfi, J. A., Billard, J. Y., 2004. An experimental study of unsteady partial cavitation. Journal of Fluids Engineering 126, 94-101.

Leroux, J.-B., Coutier-Delgosha, O., Astolfi, J. A., 2005. A joint experimental and numerical study of mechanisms associated to instability of partial cavitation on two-dimensional hydrofoil. Physics of Fluids 17 (5), 052101-21.

Lush, P., Skipp, S., 1986. High speed cine observations of cavitating flow in a duct. International Journal of Heat and Fluid Flow 7 (4), 283-290.

Mauger, C., Méès, L., Michard, M., Azouzi, A., Valette, S., 2012. Shadowgraph, schlieren and interferometry in a 2d cavitating channel flow. Experiments in fluids 53 (6), 1895-1913.

Mihatsch, M. S., Schmidt, S. J., Adams, N. A., 2015. Cavitation erosion prediction based on analysis of flow dynamics and impact load spectra. Physics of Fluids 27 (10), 103302.

Nurick, W., 1976. Orifice cavitation and its effect on spray mixing. Journal of Fluids Engineering $98(4), 681-687$.

Örley, F., Hickel, S., Schmidt, S. J., Adams, N. A., 2017. Large-eddy simulation of turbulent, cavitating fuel flow inside a 9-hole diesel injector including needle movement. International Journal of Engine Research 18 (3), 195-211.

Örley, F., Trummler, T., Hickel, S., Mihatsch, M. S., Schmidt, S. J., Adams, N. A., 2015. Largeeddy simulation of cavitating nozzle flow and primary jet break-up. Physics of Fluids 27 (8), 086101-28.

Payri, F., Bermudez, V., Payri, R., Salvador, F., 2004. The influence of cavitation on the internal flow and the spray characteristics in diesel injection nozzles. Fuel 83 (4-5), 419-431.

Petkovšek, M., Dular, M., 2013. Simultaneous observation of cavitation structures and cavitation erosion. Wear 300 (1-2), 55-64.

Pham, T., Larrarte, F., Fruman, D. H., 1999. Investigation of unsteady sheet cavitation and cloud cavitation mechanisms. Journal of Fluids Engineering 121 (2), 289-296.

Reisman, G., Brennen, C., 1996. Pressure pulses generated by cloud cavitation. In: Proc. ASME Symp. on Cavitation and Gas - Liquid Flows in Fluid Machinery and Device FED 236. American Society of Mechanical Engineers, pp. 319-328. 
Reisman, G., Wang, Y.-C., Brennen, C. E., 1998. Observations of shock waves in cloud cavitation. Journal of Fluid Mechanics 355, 255-283.

Reitz, R., Bracco, F., 1982. Mechanism of atomization of a liquid jet. The Physics of Fluids 25 (10), $1730-1742$.

Roe, P. L., 1986. Characteristic-based schemes for the euler equations. Annual Review of Fluid Mechanics 18 (1), 337-365.

Rudolf, P., Hudec, M., Gríger, M., Štefan, D., 2014. Characterization of the cavitating flow in converging-diverging nozzle based on experimental investigations. In: EPJ Web of conferences. Vol. 67. p. 02101.

Saito, Y., Sato, K., 2003. Growth process to cloud-like cavitation on separated shear layer. In: ASME/JSME 2003 4th Joint Fluids Summer Engineering Conference. pp. 1379-1384.

Sakoda, M., Yakushiji, R., Maeda, M., Yamaguchi, H., 2001. Mechanism of cloud cavitation generation on a 2-d hydrofoil. In: CAV 2001.

Sato, K., Saito, Y., 2002. Unstable cavitation behavior in a circular-cylindrical orifice flow. JSME International Journal Series B Fluids and Thermal Engineering 45 (3), 638-645.

Schmidt, S. J., 2015. A low mach number consistent compressible approach for simulation of cavitating flows. Ph.D. thesis, Technical University of Munich, Technical University of Munich.

Schmidt, S. J., Mihatsch, M. S., Thalhamer, M., Adams, N. A., 2014. Assessment of erosion sensitive areas via compressible simulation of unsteady cavitating flows. In: Kim, K.-H., Chahine, G., Franc, J.-P., Karimi, A. (Eds.), Advanced experimental and numerical techniques for cavitation erosion prediction. Springer, pp. 329-344.

Schnerr, G. H., Sezal, I. H., Schmidt, S. J., 2008. Numerical investigation of three-dimensional cloud cavitation with special emphasis on collapse induced shock dynamics. Physics of Fluids 20 (4), 040703-10.

Sou, A., Biçer, B., Tomiyama, A., 2014. Numerical simulation of incipient cavitation flow in a nozzle of fuel injector. Computers and Fluids 103 (C), 42-48.

Sou, A., Hosokawa, S., Tomiyama, A., 2007. Effects of cavitation in a nozzle on liquid jet atomization. International Journal of Heat and Mass Transfer 50 (17-18), 3575-3582.

Stanley, C., Barber, T., Milton, B., Rosengarten, G., Jun. 2011. Periodic cavitation shedding in a cylindrical orifice. Experiments in Fluids 51 (5), 1189-1200.

Stanley, C., Barber, T., Rosengarten, G., 2014. Re-entrant jet mechanism for periodic cavitation shedding in a cylindrical orifice. International Journal of Heat and Fluid Flow 50 (C), 169-176.

Stutz, B., Reboud, J. L., 1997. Experiments on unsteady cavitation. Experiments in Fluids 22 (3), 191-198.

Sugimoto, Y., Sato, K., 2009. Visualization of unsteady behavior of cavitation in circular cylindrical orifice with abruptly expanding part. In: 13th International Topical Meeting on Nuclear Reactor Thermal Hydraulics. Vol. 13. pp. N13P1156, 1-10. 
Trummler, T., Rahn, D., Schmidt, S. J., Adams, N. A., 2018. Large eddy simulations of cavitating flow in a step nozzle with injection into gas. Atomization and Sprays 28 (10), 931-955.

Wade, R., Acosta, A., 1966. Experimental observations on the flow past a plano-convex hydrofoil. Journal of Basic Engineering 88 (1), 273-282.

Wang, C., Huang, B., Wang, G., Zhang, M., Ding, N., 2017. Unsteady pressure fluctuation characteristics in the process of breakup and shedding of sheet/cloud cavitation. International Journal of Heat and Mass Transfer 114, 769-785.

Wu, X., Maheux, E., Chahine, G. L., 2017. An experimental study of sheet to cloud cavitation. Experimental Thermal and Fluid Science 83, 129-140. 